
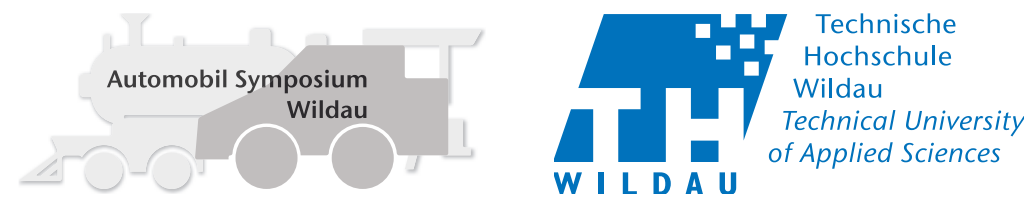

\title{
Simulation aktiver Fahrzeugsicherheitsfunktionen hochautomatisierter Fahrzeuge mit Handlungsgrundlage erweiterter Umfeldwahrnehmung mittels V2X-Kommunikation
}

\author{
Benedikt Schonlau, Daniel Hermann, Klaus Krumbiegel, David Seidel
}

doi: 10.15771/ASW_2016_4

\section{Zusammenfassung}

Die meist diskutierte und medienwirksamste Herausforderung im Automobilbereich ist aktuell das hochautomatisierte Handeln von Fahrzeugen. Fahrzeuge sollen sich durch eigenes Handeln in Teilen des Straßenverkehrs bewegen können, wie zum Beispiel bei der Fahrt auf der Autobahn oder dem Arbeitsweg mit den typischen Schwierigkeiten auf Stadt- und Landstraßen. Der Fahrer soll mit höheren Automationsgraden soweit von der Fahrverantwortung entbunden werden, dass er sich nicht mehr der Fahraufgabe widmen muss, sondern anderen Tätigkeiten nachgehen kann. Jedoch ist zu Anfang noch die Aufmerksamkeit des Fahrers gefordert, um in Situationen, die das hochautomatisierte System nicht bewältigen kann, nach einer gewissen Zeit die Fahrzeugführung zu übernehmen.

Zusätzlich besteht aktuell die Problematik der begrenzten Sensorik im Automobilbereich. Lokale Sensorik hat auf Grund der aktuellen Messverfahren wie Radar, Lidar, Kamera und Ultraschall einen begrenzten Sichtbereich und je nach Messverfahren verschiedene Einschränkungen durch äußere Umstände wie starken Regen oder flach einfallendes Sonnenlicht.

Um die Sicherheit bei der Nutzung hochautomatisierter Fahrzeuge zu erhöhen, sind ebenfalls aktive Sicherheitsfunktionen notwendig. Diese Systeme verhindern Unfälle beziehungsweise vermindern die Unfallfolgen bei unausweichlichen Notfallsituationen durch aktiven Eingriff in die Fahrzeugdynamik. Die Sicherheitsfunktionen sind jedoch stark von der Güte der Informationen über das Fahrzeugumfeld abhängig, welche aus den Eingangsdaten der Sensorik generiert werden.

Mittels V2X-Technologie, einer standardisierten Fahrzeug-zu-Fahrzeug- und Fahrzeug-zu-Infrastruktur-Kommunikation, ist ein Austausch diverser Informationen möglich. Dieser Austausch bietet neben zyklischen Daten über Position, Geschwindigkeit, Fahrzeugtyp sowie weiteren Informationen über interne Fahrzeugzustände ebenfalls die Möglichkeit, über eine detektierte Notsituation zu informieren. Diese Daten sind ebenfalls als Sensordaten zu interpretieren, deren Vorteil zum einen in einer bei weitem größeren Reichweite liegt und zum anderen Informationen zu Objekten verfügbar macht, die für lokale Sensorik durch Verdeckung oder Einschrän- kungen im Messbereich nicht erfassbar sind. Daher kann die V2X-Technologie als Erweiterung der Umfeldwahrnehmung betrachtet werden.

Die Verwendung von V2X-Technologie ermöglicht es, die Qualität und Verfügbarkeit der Informationen zum Umfeld zu erhöhen und stellt somit mehr Eingangsdaten aus unabhängigen Quellen für aktive Sicherheitsfunktionen zur Verfügung.

Zur Entwicklung von Fahrzeugfunktionen verfolgt IAV den Ansatz der szenenbasierten Funktionsentwicklung. Hierfür wird zunächst eine Idee formuliert, deren Funktionen im Folgenden in Szenen abgebildet werden. Diese Szenen stellen zum einen den Handlungsbereich der Funktion dar, zum anderen beschreiben sie die Situationen, die eine Funktion beherrschen muss. Diese Szenen dienen sowohl als Grundlage der Entwicklung des Anforderungskataloges als auch der Funktion. Mithilfe einer Abbildung der Szenen in Simulationstools lassen sich schon früh im Entwicklungsprozess die Anforderungen auf Umsetzbarkeit prüfen, die notwendige Performance der Sensorik zur Ausführung der Funktion festlegen sowie die umgesetzte Funktion im Rahmen von Softwarein-the-Loop (SiL) Simulationen testen. Nach Integration der Funktion im Fahrzeug lassen sich auf Grundlage der Szenen Testszenarien für Fahrversuche beschreiben und gegen die Anforderungen des Szenenkatalogs testen.

Das Paper soll die Vorteile von Simulation in der szenenbasierten Funktionsentwicklung anhand einer aktiven Fahrzeugsicherheitsfunktion für hochautomatisierte Fahrzeuge mittels erweiterter Umfeldwahrnehmung zeigen. Dabei werden alle Schritte von der Idee über die Anforderung und Umsetzung bis hin zu ersten Tests in der Sil-Simulation aufgezeigt. Es wird herausgestellt, welche Ergebnisse bereits im Zuge von Simulationen entstehen und wie diese verwertbar sind.

\section{Motivation}

Die Herausforderung im hochautomatisierten Fahren besteht in der Komplexität der Fahraufgabe, welche durch das System übernommen wird. Aktuelle Fahrzeugentwicklungen für Endkunden zielen dabei auf die erste Stufe der automatisierten Fahrzeugführung ab, wie in Abb. 1 als SAE Level 3 Funktion abgebildet. Im Gegensatz zu bisherigen Fahrerassistenzfunktionen, welche den 
Fahrer bei der Fahrzeugführung unterstützen, heben sich Level 3 Systeme durch die Eigenschaft der vollständigen Überwachung des Umfelds und der daraus abgeleiteten Fahrzeugdynamik ab. Wobei im englischsprachigen Raum von Bedingt-automatisierten Systemen die Rede ist, zeigt sich im Vergleich zur deutschsprachigen Definition aus Verband der Automobilindustrie (2014), mit Hochautomatisierten Systemen, eine semantische Verschiebung.

Die Anforderungen an das hochautomatisierte System sind umfangreicher, da die Funktion sich auf das gesamte System abbildet und Wechselwirkungen einzelner
Für alle Automationsstufen ist eine komplette Aufnahme der Umgebung notwendig (SAE International 2014). Aktuell werden Sensoren, die verschiedenste Messverfahren nutzen (Winner et al. 2015), wie Kamera, Radar und Ultraschallsender und -empfänger verwendet. Durch Einsatz mehrerer Sensoren ist eine redundante Beobachtung im $360^{\circ}$-Blickfeld und in Längsrichtung des Fahrzeuges über mehrere Fahrzeuglängen möglich (Winner et al. 2015). Für aktuelle Ansätze des hochautomatisierten Fahrens können diese Sensoren jedoch nicht alle Unsicherheiten abbilden, da unter anderem Verdeckung oder Reflexion das Blickfeld beeinträchtigen (Winner et

\section{Serienproduktion}

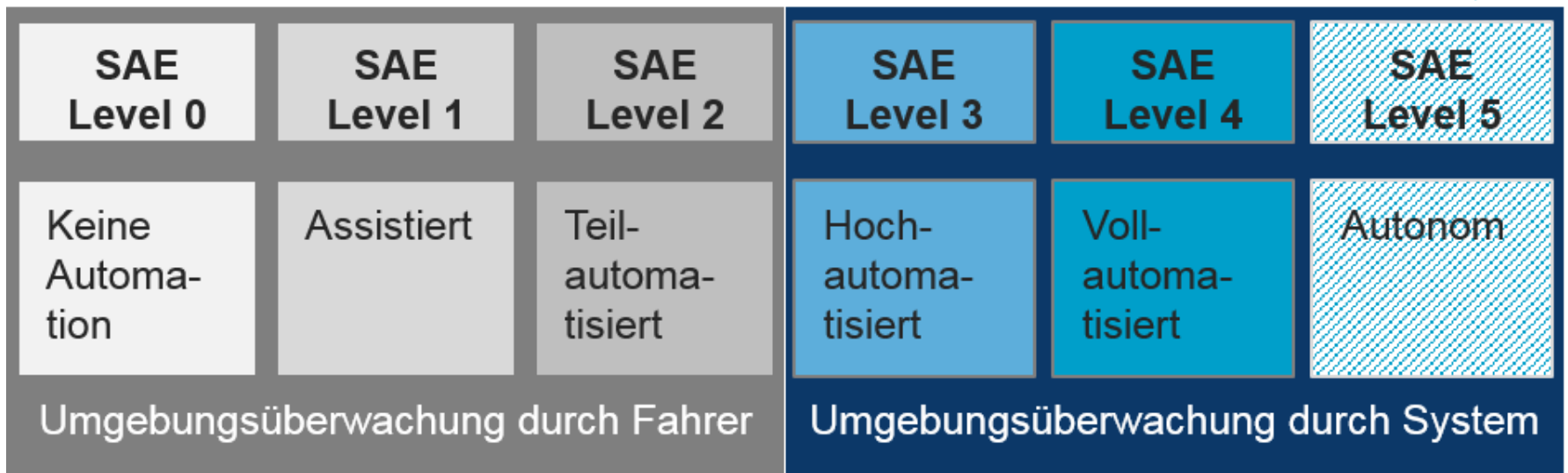

Abb. 1 Stufen der Fahrzeugautomation (SAE International 2014, Verband der Automobilindustrie 2014)

Funktionsbausteine beachtet werden müssen, im Gegensatz zu Assistenzfunktionen, welche nur Bestandteile des Gesamtsystems abbilden. Da der Fahrer für eine gewisse Zeit nicht mehr als Rückfallebene zur Verfügung steht, muss das Gesamtsystem für den vorgesehenen Einsatzzweck sicher gestaltet werden und somit bis zur Übergabe jede Situation behandeln können.

Das Paper zeigt auf, wie durch Simulation in Kombination mit szenenbasierter Funktionsentwicklung die Gewährleistung von Sicherheit und Effizienz der Entwicklung gesteigert werden kann.

\section{Stand der Technik}

Das hochautomatisierte Fahren übernimmt die Fahraufgabe für den Fahrer beschränkt auf bestimmte Einsatzzwecke, wie die Fahrzeugführung im Stau auf der Autobahn. Höhere Automatisierungsstufen, wie in SAE International (2014) beschrieben, sehen eine Übernahme der Fahrzeugführung in den meisten Einsatzszenarien vor, bezeichnet durch vollautomatisierte Systeme beziehungsweise als SAE Level 4. Autonome Systeme, SAE Level 5, sollen die komplette Fahraufgabe in allen Situationen übernehmen, wobei ein Fahrzeugführer als Rückfallebene nicht mehr vorgesehen ist. Im Folgenden werden Systeme des SAE Level 3, hochautomatisierte Systeme, betrachtet. al. 2015).

Um die Umfeldwahrnehmung zu erweitern, wird die Technologie der Fahrzeug-zu-Fahrzeug- beziehungsweise Fahrzeug-zu-Infrastruktur-Kommunikation verwendet. Die mit Vehicle2X (V2X) bezeichnete Technologie basiert auf dem IEEE 802.11p Standard und soll Reichweiten bis zu $1000 \mathrm{~m}$ abdecken (Winner et al. 2015). Mittels Multi-Hopping werden Nachrichten zwischen den Knoten des Netzwerkes weitergeleitet, wodurch noch größere Reichweiten erreicht werden (Winner et al. 2015). So ist auch eine größere Abdeckung in schwierigen Gebieten wie Städten möglich, trotz der Reichweiten begrenzenden Einflüsse wie Reflexion oder Verdeckung. Der Einsatz zyklischer sowie auch ereignis- beziehungsweise bereichs-gesteuerter Nachrichten ermöglicht die Übertragung des Fahrzeugzustandes per Cooperative Awareness Messages (CAM) als auch die Übermittlung besonderer Ereignisse per Decentralized Environmental Notification Message (DENM) (Winner et al. 2015). Die V2X-Kommunikation kann als weiterer Sensor, neben den der lokal begrenzten Sensorik, interpretiert werden (Schonlau et al. 2015).

Der Entwicklungsprozess hochautomatisierter Fahrzeugfunktionen wird im V-Modell durchgeführt, wobei, je nach Reifegrad, wie Vorentwicklung oder Serienentwicklung, dieser Prozess mehrmals durchlaufen werden muss (Schömann 2012). Besonders für Fahrzeugfunktio- 
nen mit Umfeldsensorik eignet sich die Anwendung szenenbasierter Funktionsentwicklung. Der Vorteil besteht in der Abbildung der gewünschten Funktion in Szenarien, welche die Interaktion zwischen Sensorik, Umgebung und resultierender Aktorik darstellen (Schonlau et al. 2014).

Aktuell wird die szenenbasierte Funktionsentwicklung genutzt, um frühzeitig in Projekten die Funktionalität in Fahrsituationen durch Simulation abzubilden sowie später zu testen. Der Vorteil liegt in der Kosten- und Zeitersparnis, da Versuchsfahrten durch Simulation reduziert und schneller durchgeführt sowie die Wechselwirkungen verschiedenster Kombinationen von Sensoren oder Aktoren getestet werden können.

Mit Hilfe der Funktionsspezifikation lässt sich der Funktionsumfang in Szenen abbilden. Die Szenen helfen im Folgenden der Erstellung der Anforderung an die Funkti- des Simulationstools, Scene Suite Connect, lassen sich zur Laufzeit der Simulation die Daten der Sensoren auswerten, Funktionalitäten implementieren und die Dynamik der Objekte anpassen. Mit Erstellung der Szenarien aus der Spezifikation in Scene Suite lassen sich diese im kompletten folgenden Entwicklungsprozess zur Simulation des angestrebten Verhaltens, der Simulation des Verhaltens nach Anforderung sowie zum Testen des Softwarecodes nutzen.

3. Simulation für sicherheitsrelevante Funktionen im hochautomatisierten Fahren

Besonders für sicherheitsrelevante Funktionen ist Simulation eine geeignete Methode, um diese auf Vollständigkeit zu prüfen und Funktionstests für kritische Situationen durchzuführen. Die Anforderungen an den

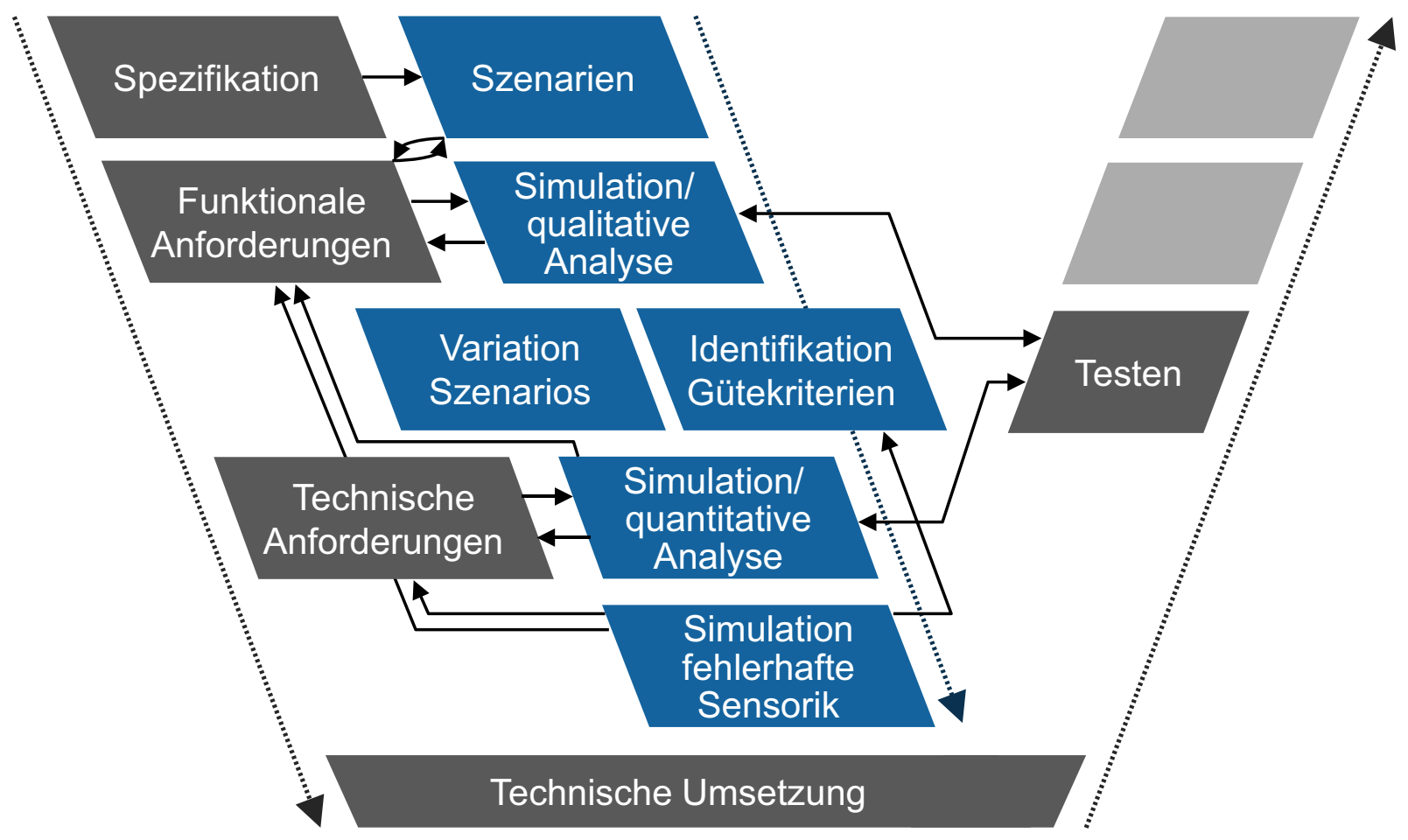

Abb. 2 Simulation in szenenbasierter Funktionsentwicklung

on. Durch Analyse der Szenarien mit abgebildetem Funktionsverhalten können Realisierbarkeit des Verhaltens sowie Anforderungen an Sensorik und Aktorik geprüft werden. Die Szenarien bilden im Folgenden, neben den Anforderungen, die Grundlage für die Softwareentwicklung, worauf diese wiederum durch Software-in-the-Loop Simulation der Szenarien getestet werden kann.

Zur Umsetzung der Simulation kann das Tool Scene Suite genutzt werden. Das Tool stellt dafür Szenarien auf Grundlage der dynamischen Vorgaben für Objekte dar und berechnet für jeden Zeitschritt die Dynamik der Objekte neu. Des Weiteren können Sensoren und Aktoren mit den Objekten genutzt werden, deren Verhalten beziehungsweise Ausgabe ebenfalls für jeden Zeitschritt generiert werden. Durch Verwendung einer Schnittstelle
Entwicklungsprozess für hochautomatisierte Fahrzeugfunktionen sind um ein vielfaches höher als für Assistenzfunktionen der SAE Level 1 und 2. Da der Fahrer nicht mehr kurzfristig als Rückfallebene zur Verfügung steht und die Funktion auf das Gesamtsystem und seine Umgebung wirkt, ist ein höheres Maß einer sicheren Funktionalität zu gewährleisten.

Der Prozess zur Simulation in der szenenbasierten Funktionsentwicklung ist in Abb. 2 dargestellt und beispielhaft an einer Funktion in Kapitel 4 angewandt. Die Funktion für hochautomatisierte Fahrzeuge wird zu Anfang des V-Prozesses spezifiziert. Mit Hilfe der Spezifikation können viele Funktionalitäten in Szenarien abgebildet werden. Für die angestrebte Methodik sind an diesem Punkt bereits die Parameter zu identifizieren, welche das 
Szenario in Bezug auf den Teil der abgebildeten Funktion signifikant beeinflussen.

Auf Grundlage der Spezifikation und den definierten Szenarien werden die funktionalen und technischen Anforderungen entwickelt, womit sich weitere Szenarien ergeben können. Mit Verfügbarkeit der Szenarien können die funktionalen Anforderungen bereits in abstrakter Form umgesetzt und per Simulation getestet werden. Diese Umsetzung passiert absolut unabhängig vom Softwareentwicklungsprozess und kann parallel oder zuvor geschehen. Eine qualitative Analyse der simulierten Szenarien identifiziert nicht ausreichende oder zu restriktive Anforderungen beziehungsweise Unzulänglichkeiten des Systems in Bezug auf die geforderte Funktion.

Zur quantitativen Analyse der Szenarien sind diese zu variieren und Gütekriterien zur Bewertung einzuführen. Die Szenarien variiert man anhand ihrer zuvor festgelegten Parameter und erhält eine Vielzahl an Szenarien zum Prüfen. Mit den Gütekriterien lassen sich die Performance der Funktion und technische Anforderungen für das System bestimmen. Die Simulation kann im ersten Schritt unter perfekten Bedingungen durchgeführt werden, um das rein funktionale Verhalten zu analysieren und die Gütekriterien zu ermitteln. Mit Bewertung der Gütekriterien lässt sich mögliches funktionales Fehlverhalten bestimmen und entsprechend in den funktionalen Anforderungen anpassen. Mit schrittweiser Verschlechterung von Systembestandteilen, welche die Funktion direkt oder indirekt beeinflussen, wie zum Beispiel Sensoren und deren Daten, und einer entsprechenden Simulation der variierten Szenarien, ergeben sich neue Werte für die Gütekriterien. Im Vergleich zu den Gütekriterien der perfekten Bedingungen können Schlüsse auf die Performance der technischen Bestandteile gezogen, Randbedingungen für Umgebungseinflüsse festgelegt sowie die Qualität der Gütekriterien bestimmt werden. Mit jeder Iteration im Prozess durch Erweiterung der Anforderungen oder Korrektur der Gütekriterien lässt sich bereits auf Anforderungsseite eine gewisse Qualität der Funktion gewährleisten und der Aufwand durch Testfahrten sowie daraus folgende Anpassungen der Anforderungen minimieren.

Auf Grundlage der Anforderungen kann nun das technische System im Sinne von Hardware und Software umgesetzt werden. Auf Basis der Szenarien und identifizierten Gütekriterien besteht weiterhin die Möglichkeit die Software in Software-in-the-Loop Tests zu verifizieren. Ergeben sich aus der Umsetzung weitere Anforderungen, welche durch Simulation auftreten, müssen diese entsprechend angepasst werden und der Prozess wird wiederholt.

4. Anwendung auf eine Fahrzeugsicherheitsfunktion für hochautomatisierte Fahrzeuge

Im Folgenden wird die Methodik am Beispiel einer Fahrzeugsicherheitsfunktion für hochautomatisierte Fahrzeuge mit erweiterter Umfeldwahrnehmung angewandt.
Neben der Unfallverhinderung ist die Minimierung von Unfallrisiken durch Sicherheitsfunktionen ein zusätzliches Ziel für hochautomatisierte Fahrzeuge.

\subsection{Spezifikation der Funktion und Szenario}

Die Funktion bildet eine automatisierte Notbremsung für hochautomatisierte Fahrzeuge auf der Autobahn ab. Die Funktion zur Längsregelung führt das Fahrzeug immer im 2 s Abstand zum vorausfahrenden Fahrzeug. Optional ist ein 1,5 s Abstand vorgesehen. Die Sicherheitsfunktion soll für diesen Fall eine Kollision mit allen vorausfahrenden Fahrzeugen in der eigenen Spur so komfortabel wie möglich verhindern. Folgende synthetische Sensoren sind nutzbar: Radar, Blickfeld $250 \mathrm{~m}$ bei $18^{\circ}$ Öffnungswinkel, und ein Lidar, Blickfeld $120 \mathrm{~m}$ bei $145^{\circ}$ Blickfeld unter der Annahme idealer Messbedingungen.

Die Analyse der Spezifikation führt zu diversen Szenarien: der einfachen Folgefahrt, Start und Stopp, stark bremsendes vorausfahrendes Fahrzeug, Objekte auf der eigenen Fahrspur, Fahrzeuge die auf die Spur einscheren beziehungsweise ausscheren und etliche weitere. Für die folgende Analyse wird ein kritisches Ereignis näher betrachtet: das vorausfahrende Fahrzeug weicht kurzfristig zwei kollidierten, stehenden Fahrzeugen auf der eigenen Fahrbahn aus. Das Szenario ist von folgenden Parametern abhängig:

- der Eigengeschwindigkeit ( $v E g o)$

- dem Abstand zum vorausfahrenden Fahrzeug (dEgoToTarget)

- die Distanz des vorausfahrenden Fahrzeugs zu den kollidierten Fahrzeugen zu welchem der Spurwechsel startet und

- die Zeit des Spurwechsels.

Für die folgende Betrachtung werden die ersten beiden Parameter variiert,

$$
v E g o=\frac{(80: 160,20)[\mathrm{km} / \mathrm{h}]}{d \text { EgoToTarget }}=(1.5: 2.0,0.5)[\mathrm{s}]
$$

für die Zeit des Spurwechsels werden $2 \mathrm{~s}$ angenommen und die Entfernung resultiert aus einer knappen Vermeidung der Kollision. Somit ergeben sich 10 Variationen, welche jeweils aus zwei Teilen bestehen, der klassischen Folgefahrt für $2 \mathrm{~s}$ und dem Ausweichmanöver für $2 \mathrm{~s}$.

\subsection{Funktionale Anforderung und qualitative Analyse}

Die funktionale Anforderung an die automatische Notbremsung wäre in diesem Fall, eine Kollision mit den beiden kollidierten Fahrzeugen zu verhindern und hinter diesen zum Stehen zu kommen. Für die Detektion aller Objekte stehen vorerst nur Radar und Lidar zur Verfügung. 
In der Umsetzung analysiert die Bremsfunktion alle Fahrzeuge auf der eigenen Spur und fordert eine Bremsverzögerung an, um den notwendigen Abstand zu halten als auch hinter stehenden Fahrzeugen in den Stillstand zu bremsen, im Idealfall $5 \mathrm{~m}$ hinter dem Objekt.

Die qualitative Analyse zeigt, dass der unkritischste Fall eine Verzögerung von $4.2 \mathrm{~m} / \mathrm{s}^{2}$ erfordert und die Kollision vermieden wird. Jedoch kommt es im kritischsten Fall

$$
v E g o=\frac{80 \mathrm{~km} / \mathrm{h}}{d E g o \text { ToTarget }}=2 \mathrm{~s}
$$

trotz vollster Verzögerung von $8 \mathrm{~m} / \mathrm{s}^{2}$ zur Kollision. Die Verdeckung durch das vorausfahrende Fahrzeug ermög-

$$
v E g o=\frac{160 \mathrm{~km} / \mathrm{h}}{d E g o \text { ToTarget }}=1.5 \mathrm{~s}
$$

licht erst eine späte Detektion der Gefährdung.

Die funktionale Anforderung zu ändern, würde in diesem Fall zu einem bei weitem höheren Sicherheitsabstand zu vorausfahrenden Fahrzeugen führen, was jedoch zu einem ungewohnten Fahrgefühl für Fahrer und andere Verkehrsteilnehmer führt.

\subsection{Variation Szenen, Identifikation Gütekriterien und quantitative Analyse}

Variiert man die Szenarien nach den zuvor identifizierten Parametern, lässt sich das Funktionsverhalten im gegebenen Rahmen beobachten. Zur Optimierung der Anforderung sind folgende Gütekriterien eingeführt: Keine Kollision, Verzögerung von $0.5-3 \mathrm{~m} / \mathrm{s}^{2}$ als Komfortbremsung, $3-5 \mathrm{~m} / \mathrm{s}^{2}$ als Gefahrenbremsung und größer als $5 \mathrm{~m} / \mathrm{s}^{2}$ als Notbremsung. Ziel ist es, entsprechend häufig keine Kollision zu haben sowie die meiste Zeit der Verzögerung im komfortablen Bereich zu sein.

Tab. 1 Gütekriterien summiert für alle 10 Variationen im Vergleich vor und nach der Optimierung

\begin{tabular}{|c|c|c|c|}
\hline & $\begin{array}{c}\text { Initiales } \\
\text { Funktions- } \\
\text { verhalten }\end{array}$ & $\begin{array}{c}\text { Funktionsver- } \\
\text { halten nach } \\
\text { Optimierung }\end{array}$ & $\begin{array}{c}\text { Funktionsver- } \\
\text { halten nach } \\
\text { Optimierung } \\
\text { mit Fehlern }\end{array}$ \\
\hline Kollision $[\mathrm{n}]$ & 4 & 0 & 0 \\
\hline $0.5-3 \mathrm{~m} / \mathrm{s}^{2}[\mathrm{~s}]$ & 1.98 & 20.58 & 4.36 \\
\hline $3-5 \mathrm{~m} / \mathrm{s}^{2}[\mathrm{~s}]$ & 6.22 & 55.42 & 35.52 \\
\hline$>5 \mathrm{~m} / \mathrm{s}^{2}[\mathrm{~s}]$ & 34.26 & 9.5 & 30.18 \\
\hline
\end{tabular}

Die in Tab. 1 aufgezeichneten Werte für die Gütekriterien zeigen ein kritisches Systemverhalten mit 4 Kollisionen und sehr häufiger Notbremsung. Dies ist auf Grundlage der qualitativen Analyse auf den begrenzten Sichtbereich der Sensorik zurückzuführen.

Durch zusätzliche Integration synthetischer V2X-Technologie lassen sich jedoch weitere Daten in Form von CAMs und DENMs empfangen. Dadurch sieht das Fahrzeug rechtzeitig die Gefahrenquelle und eine frühere Einlei- tung der Bremsung ist möglich. Als Empfangsbereich wird ein Umkreis von $200 \mathrm{~m}$ angenommen. Dieser ist auf Grund der begrenzten Simulationszeit ausreichend, da keine Szene eine weitere Entfernung zu den Objekten beinhaltet.

Die Ergebnisse in Tab. 1 zeigen, dass sich das Fahrzeug im Rahmen der Variation unter idealen Bedingungen ausreichend sicher verhält. Es gibt keine Kollisionen mehr und die Komfortbremsung kommt entsprechend häufiger vor.

\subsection{Anpassung der Anforderung und Simulation un- ter schlechten Bedingungen}

Mit den Ergebnissen der qualitativen und quantitativen Analyse ergibt sich die weitere technische Anforderung V2X-Technologie zu nutzen, um eine sichere Funktionalität zu gewährleisten.

Mit Beeinträchtigung der synthetischen V2X-Technologie durch schlechte Performance kann die Güte der Technik in Kombination mit der Funktion bestimmt werden und so eine Mindestanforderung an die Systembestandteile bestimmen oder durch funktionale Anpassung bekannte Schwächen von Systembestandteilen ausgleichen. Entsprechend ist der Versand der CAM und DENM Nachrichten auf $1 \mathrm{~Hz}$ verringert und die erste Meldung frühestens nach $1 \mathrm{~s}$ Simulationslaufzeit.

Die Auswertung von Tab. 1 ergibt trotz der Schwächen ein ausreichend sicheres Funktionsverhalten. Kollisionen werden weiterhin verhindert, nur die Verzögerungen sind stärker. Die Diagramme in den Abb.3-5 zeigen das Funktionsverhalten der drei Simulationen für den schwierigsten Fall mit einer Geschwindigkeit von $160 \mathrm{~km} / \mathrm{h}$ und einem Abstand zum vorausfahrenden Fahrzeug von $1.5 \mathrm{~s}$.

Die Auswertung der Diagramme zeigt, dass das initiale Verhalten bei etwa $6.5 \mathrm{~s}$ zur Kollision führt, trotz maximal verfügbarer Verzögerung. Ungeachtet zusätzlicher Fehler für die optimierte Funktion wird eine Kollision verhindert. Der verzögerte Empfang der CAM bzw. DENM ist in Abb. 5 durch die verzögerte Reaktion nach $1 \mathrm{~s}$ zu sehen.

\section{Fazit und Ausblick}

Die frühzeitige Verwendung von Simulation im Entwicklungsprozess insbesondere in Kombination mit szenenbasierter Funktionsentwicklung lässt rechtzeitig Schlüsse über die Qualität der Anforderungen zu und verringert den Einsatz aufwändiger Testverfahren. Des Weiteren werden mögliche Änderungen der Anforderungen im fortgeschrittenen Prozess minimiert. Durch Simulation über eine Vielzahl von Szenarien und der Variation dieser, lassen sich Unzulänglichkeiten funktionaler oder technischer Bestandteile über eine große Bandbreite an Einsatzzwecken identifizieren. Vor allem für sicherheitsrelevante Funktionen, welche meist eine sicherheitskri- 
Distanz zu kollidierten Objekten
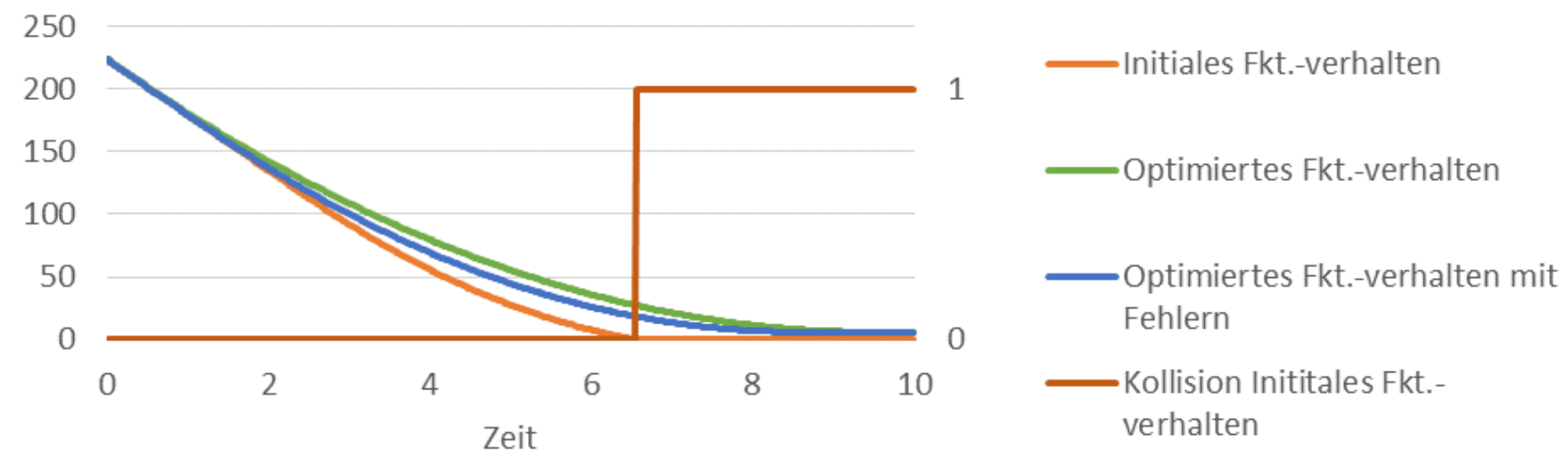

Abb. 3 Vergleich der Funktionsverhalten bezüglich der Distanz zu kollidierten Objekten

Geschwindigkeitsprofil

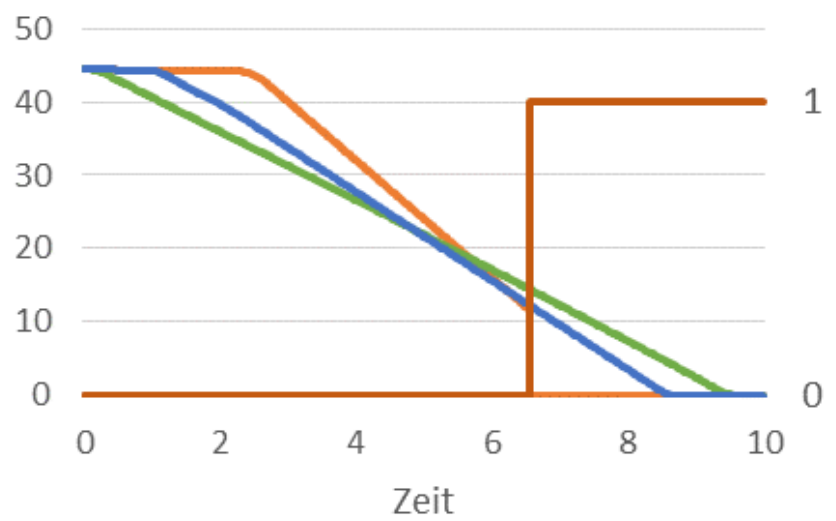

Abb. 4 Vergleich Geschwindigkeit der einzelnen Funktionsverhalten

tische Situation voraussetzen, ist Simulation eine gute Alternative um Funktionalität zu testen und Sicherheit zu gewährleisten. Mit Hilfe der Automation der Prozessschritte ist die Anzahl an Szenarien und ihrer Variation unbegrenzt, jedoch der Aufwand der folgenden quantitativen Analysen recht gering. Durch Anwendung von Simulation nach diesem Prozess in einer geeigneten Werkzeugkette lassen sich Kosten und Zeitaufwände senken sowie für ein großes Spektrum an Einsatzzwecken sichere Funktionen gewährleisten.

Die Ergebnisse beziehungsweise identifizierten Szenarien, Variationen und Gütekriterien lassen sich in späteren Prozessschritten als zusätzlich Testgrundlage nutzen, zum Beispiel durch Anwendung einer ähnlichen Methodik in SiL-Tests für die umgesetzte Software. Die Anforderungen an die Simulation sind jedoch entsprechend höher, durch eine notwendige Realitätsnähe.

Der Einsatz von V2X-Technologie ermöglicht das hochautomatisierte Fahren in Situationen, in welchen lokale Sensorik durch systematische Einschränkungen oder äußere Einflüsse an ihre Grenzen stößt. V2X kann als zusätzlicher Sensor interpretiert werden und liefert neben dynamischen Informationen zu Objekten, weitere Daten wie zum Beispiel Positionen von sicherheitskritischen Orten.

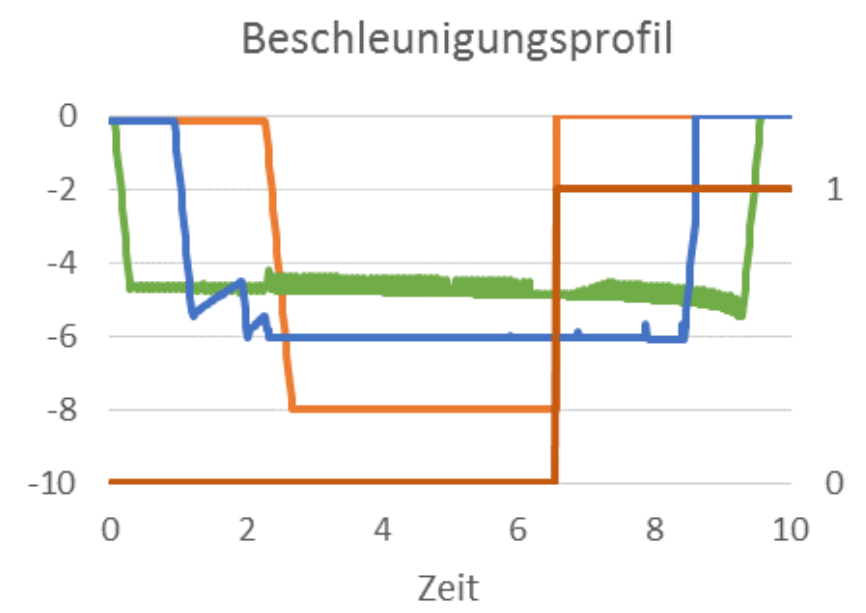

Abb. 5 Vergleich Beschleunigung der einzelnen Funktionsverhalten

\section{Literatur}

SAE International (2014) Automated Driving. Levels of Driving Automation Are Defined in New SAE International Standard J3016. http://www.sae.org/servlets/pressRoom?OBJECT_TYPE=PressReleases\&PAGE=showRelease\&RELEASE_ ID=2715. Accessed 17 Feb 2016

Schömann SO (2012) Produktentwicklung in der Automobilindustrie. Managementkonzepte vor dem Hintergrund gewandelter Herausforderungen. Gabler Research: Schriften zur Unternehmensentwicklung. Gabler, Wiesbaden. ISBN: 978-3-8349-2874-0. doi: 10.1007/978-3-8349-6673-5

Schonlau B, Grimm S, Seidel D, Sternberg M (2015) Automatische Notfallbremsung zur Unfallschwereminderung durch erweiterte Umfeldwahrnehmung mittels C2X. Praktische Umsetzung und Ergebnisse. In: VDI (ed) VDI Fahrzeugsicherheit, Berlin

Schonlau B, Rudolf L, Wenzel P, Krumbiegel K (2014) Softwarein-the-Loop Tests for the New Generation of Driver Assistance Systems and Active Safety Functions. In: IAV (ed) Sixth Conference on Simulation and Testing for Automotive Electronics, Berlin

Verband der Automobilindustrie (2014) Automatisiertes Fahren. Stufen des automatisierten Fahrens. https://www.vda. de/de/themen/innovation-und-technik/automatisiertes-fahren/automatisiertes-fahren.html. Accessed 17 Feb 2016 
Winner H, Hakuli S, Lotz F, Singer C (eds) (2015) Handbuch Fahrerassistenzsysteme. Grundlagen, Komponenten und Systeme für aktive Sicherheit und Komfort, 3rd edn. ATZMTZ-Fachbuch. Springer Vieweg, Wiesbaden. ISBN: 978-3658-05733-6. doi: 10.1007/978-3-8348-8619-4

\section{Autoren}

Benedikt Schonlau

IAV GmbH

Benedikt.Schonlau@iav.de

Daniel Hermann

IAV GmbH

Daniel.Hermann@iav.de

Dr. Klaus Krumbiegel

IAV GmbH

Klaus.Krumbiegel@iav.de

David Seidel

IAV GmbH

David.Seidel@iav.de

(C) $9=\begin{aligned} & \text { Dieser Beitrag ist unter der } \\ & \text { Creative-Commons-Lizenz } \\ & \text { CC BY-NC-ND lizensiert. }\end{aligned}$ 